\title{
Cost, Effectiveness, and Value
} How to Judge?

Michael D. Rawlins

MD

London School of Hygiene and Tropical Medicine, London, England.
Corresponding Author: Michael D. Rawlins, MD, London School of Hygiene and Tropical Medicine, Keppel St, London WCIE 7HT, England (michael.rawlins@nice .org.uk).
Universal health coverage is a global aspiration supported by both the World Health Organization ${ }^{1}$ and the United Nations. ${ }^{2}$ The World Health Organization has defined universal health coverage as ensuring that "all people obtain the health services they need without suffering financial hardship when paying for them."1 The UN resolution supporting universal health coverage specifically avoided defining a particular type of health financing system, but called on member states "to ensure that health financing systems evolve so as to avoid significant direct payments at the point of delivery."2

The resources available to finance health care in individual countries are closely correlated with the wealth of those countries. ${ }^{3}$ Richer countries spend more on health care than poorer ones; and there is a remarkably close correlation between a country's gross domestic product and its expenditure on health care, even when public and private resources for health care are combined. ${ }^{3}$ No country, however, is sufficiently resourced to be able to meet all the aspirations of universal health coverage. Priorities have to be made in the trade-off between the cost of an intervention (whether diagnostic or therapeutic) and the additional benefit associated with the intervention.

The relationship between cost and effectiveness can be depicted ${ }^{3}$ as a cost-effectiveness plane (Figure). Interventions that are more expensive but less effective (quadrantAin the Figure) will obviously be rejected. Those interventions that are less expensive but also less effective (quadrant B) would be more challenging for decision makers but in reality are unusual. Interventions that are more effective but less expensive (quadrant C) should be readily adopted, but are relatively uncommon. The more expensive and more effective interventions in quadrant $D$ pose real problems for decision makers in both developed and developing countries. How much in the way of additional costs, in relation to the anticipated increase in effectiveness, can a health care system afford without displacing other more effective interventions?

In attempting to answer this question, this Viewpoint focuses on pharmaceutical agents, considering the concerns involving the high costs of some of these highly effective products. However, the same principles apply equally to devices, diagnostic techniques, and other health care delivery systems.

\section{Costs}

The costs of an intervention are, in theory, easy to define. They include the acquisition costs of the intervention, the costs of its administration, and the savings (sometimes called cost offsets) that accrue from use of an intervention. The true acquisition costs of new pharmaceuticals are often opaque because of commercially confidential discounts that payers in many developed countries negotiate and are often substantially less than the list price. In developing countries, payers should similarly negotiate for lower prices for products from developed countries.

\section{Effectiveness}

The evidence of the effectiveness of an intervention might seem easy to define. Regulatory authorities, for example, rigorously assess the evidence of clinical effectiveness of new pharmaceutical agents before they are marketed. But, again, there are difficulties.

First, evidence for the clinical effectiveness at the time a new product is marketed will generally have been based on placebo-controlled studies, active comparatorcontrolled studies, or a combination of the 2 . The problem is that this approach ignores the fact that there are often substantial differences within and between different health care systems in their accepted standards of care and hence the relevant comparators. ${ }^{4}$ Analytic techniques such as indirect comparisons and network analyses ${ }^{3}$ can help overcome these difficulties but require considerable expertise in application.

The second problem in the evaluation of clinical effectiveness is that methods of determining effectiveness are often disease or condition specific. Organizations such as the American Society of Clinical Oncology, the European Society for Medical Oncology, and the Memorial Sloan Kettering Cancer Center all use approaches that evaluate specific response rates or survival rates ${ }^{5}$ among treated patients. Even though these techniques provide evidence of effectiveness in specific conditions, they are of virtually no use to organizations seeking to compare the costs and benefits of treating one condition compared with another. For example, how are payers expected to compare the value of a new treatment for breast cancer with a new treatment for schizophrenia if the benefits are expressed in totally different ways?

An alternative approach seeks to assess effectiveness in a manner that can be used to make appropriate comparisons between different conditions. Groups such as the American College of Cardiology, the Institute for Clinical Effectiveness Research in the United States, and the National Institute for Health and Clinical Effectiveness in the United Kingdom use metrics such as the quality-adjusted life-years (QALYs) gained so the clinical benefits of interventions can be compared across disease states. In this way, payers can negotiate acquisition prices as well as make informed decisions about relative priorities. The use of QALYs is beneficial for policy makers more generally as well as to developers of clinical guidelines. 
Figure. Cost-effectiveness Plane for Assessing a New Intervention vs a Comparator

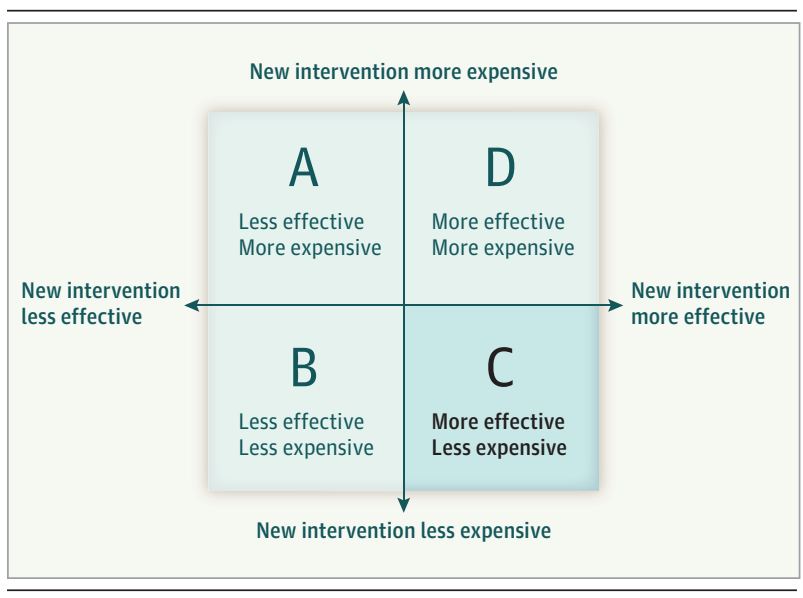

Values and Judgments

Decisions about whether to recommend or adopt particular interventions also require making value judgments, which generally involve scientific and social perspectives. ${ }^{6}$

Scientific value judgments are concerned with (1) considering how reliable the evidence base is; (2) whether the results are generalizable to the population likely to be treated; (3) whether subgroup analyses are appropriate; and (4) whether the changes in quality of life (an essential component of estimating the QALYs) have been appropriately captured. Contrary to popular belief, these are all judgments. No clinical trial is ever perfect in that all have limitations and deficiencies. Do these invalidate the results? Are the findings generalizable to use in the real world?

In a misguided attempt to avoid making such scientific judgments, some resort to applying hierarchies of evidence, ${ }^{7}$ many of which have been published during the past 30 years. All hierarchies assign randomized clinical trials at the highest evidence levels, with observational studies at lower levels. However, the ordering of evidence in this way is inappropriate because it is not the methods per se that are important but whether the methods are fit for purpose. As a result, confidence of the benefits of penicillin for the treatment of pneumonia ${ }^{7}$ (originally based on evidence from observational studies) is no less secure than confidence in the benefits of trastuzumab in the early treatment of breast cancer based on randomized clinical trials.

Decision makers or payers also have to make social value judgments based on social considerations rather than biomedical science. ${ }^{6}$ What threshold should be used to distinguish between cost-effective and cost-ineffective interventions? Should added weight be given to the severity of the underlying condition in deciding whether an intervention should be provided by a health care system? Even when an intervention appears to be costeffective with a cost of $\$ 50000$ per QALY, the overall budgetary effect may be substantial if large numbers of patients are likely to seek that intervention. An important issue becomes what threshold should be applied in considering the affordability of an intervention for the health care system as a whole?

Moreover, how should the tensions between utilitarianism (ie, the greatest good for the greatest number) be resolved against egalitarianism (ie, treating everyone equally)? For example, how should health inequalities based on socioeconomic and ethnic factors be incorporated into decision making?

All of these matters that have to be especially considered in decision making by health care systems for which significant financial contributions are made from public funds. In some countries, politicians and governments make these determinations, whereas in other countries, the responsibility is largely handed over to arms-length bodies such as the National Institute for Health and Clinical Effectiveness in the United Kingdom. It is unclear to observers of the US health care system who in the United States is making these decisions.

\section{Conclusions}

The need for priority setting in the global search for universal health coverage is clear. How this is undertaken is a regional or national endeavor that depends as much on the prevailing political and socioeconomic circumstances as it does the underlying biomedical science. But ultimately, all health care systems must embrace both the opportunities and the challenges that are involved.

\section{ARTICLE INFORMATION}

Conflict of Interest Disclosures: The author has completed and submitted the ICMJE Form for Disclosure of Potential Conflicts of Interest. Dr Rawlins reported being the founding chair of the National Institute of Health and Care Excellence and serving as chair from 1999 to 2013.

Additional Contributions: I am grateful to Steven D. Pearson, MD, MSc (Institute for Clinical Effectiveness Research), for his helpful comments on earlier drafts of the article.

\section{REFERENCES}

1. World Health Organization. Research for Universal Health Care Coverage. Geneva, Switzerland: World Health Organization; 2013.

2. UN General Assembly. A/67/L.36: global health and foreign policy. http://www.un.org/ga/search /viewm_doc.asp?symbol=A/67/L.36. Accessed September 15, 2016.

3. Rawlins MD. Evidence, Therapeutics and Decision-Making. London, England: Hodder; 2011

4. Sanders GD, Neumann PJ, Basu A, et al. Recommendations for conduct, methodological practices, and reporting of cost-effective analyses: Second Panel on Cost-Effectiveness in Health and Medicine. JAMA. 2016;316(10):1093-1103.

5. Chandra A, Shafrin J, Dhawan R. Utility of cancer value frameworks for patients, payers, and physicians. JAMA. 2016;315(19):2069-2070.

6. Rawlins MD, Culyer AJ. National Institute for Clinical Excellence and its value judgments. BMJ. 2004;329(7459):224-227.

7. Rawlins M. De testimonio: on the evidence for decisions about the use of therapeutic interventions. Lancet. 2008;372(9656):2152-2161. 